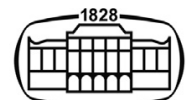

AKADÉMIAI KIADÓ

\title{
The neural correlates of delay discounting in obesity and binge eating disorder
}

Journal of Behavioral Addictions

10 (2021) 3, 498-507

\section{Special Section on} Overweight, obesity and eating disorders in relation to addiction

\section{DOI:}

10.1556/2006.2021.00023 (c) 2021 The Author(s)

\section{FULL-LENGTH REPORT}

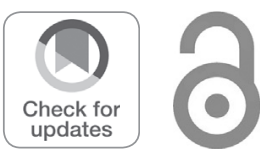

${ }^{\dagger}$ Romina Miranda-Olivos and Trevor Steward share first authorship.

${ }^{*}$ Corresponding author.

E-mail: csoriano@idibell.cat

** Corresponding author.

E-mail: ffernandez@bellvitgehospital. cat
ROMINA MIRANDA-OLIVOS ${ }^{1,2,3 \dagger}$, TREVOR STEWARD ${ }^{4 \dagger}$, IGNACIO MARTÍNEZ-ZALACAÍN ${ }^{1,3}$, GEMMA MESTRE-BACH ${ }^{5}$, ASIER JUANEDA-SEGUII ${ }^{1,3,6}$, SUSANA JIMÉNEZ-MURCIA ${ }^{1,2,3}$, JOSÉ A. FERNÁNDEZ-FORMOSO ${ }^{2}$, NURIA VILARRASA ${ }^{7,8}$, MISERICORDIA VECIANA DE LAS HERAS ${ }^{9}$, NURIA CUSTAL ${ }^{1}$, NURIA VIRGILI ${ }^{7}$, RAFAEL LOPEZ-URDIALES ${ }^{7}$, JOSÉ M. MENCHÓN ${ }^{1,3,6}$, ROSER GRANERO ${ }^{2,10}$,

CARLES SORIANO-MAS ${ }^{1,6,10 *}$ (๑) and FERNANDO FERNANDEZ-ARANDA ${ }^{1,2,3 * *} \odot$

\footnotetext{
${ }^{1}$ Department of Psychiatry, Bellvitge University Hospital-IDIBELL, C/Feixa Llarga s/n, 08907, Barcelona, Spain

${ }^{2}$ Ciber Fisiopatología Obesidad y Nutrición (CIBERObn), Instituto Salud Carlos III, C/Monforte de Lemos 3-5, 28029 Madrid, Spain

${ }^{3}$ Department of Clinical Sciences, School of Medicine, University of Barcelona, C/Feixa Llarga s/n, 08907, Barcelona, Spain

${ }^{4}$ Melbourne School of Psychological Sciences, Faculty of Medicine, Dentistry and Health Sciences, University of Melbourne, Parkville, VIC, 3010, Australia

${ }^{5}$ Universidad Internacional de La Rioja, La Rioja, Spain

${ }^{6}$ Ciber Salud Mental (CIBERSAM), Instituto Salud Carlos III, C/Monforte de Lemos 3-5, 28029 Madrid, Spain

${ }^{7}$ Department of Endocrinology and Nutrition, University Hospital of Bellvitge-IDIBELL, C/Feixa Llarga s/n, 08907, Barcelona, Spain

${ }^{8}$ CIBERDEM-CIBER de Diabetes y Enfermedades Metabólicas Asociadas, Instituto de Salud Carlos III, C/Monforte de Lemos 3-5, 28029 Madrid, Spain

${ }^{9}$ Neurology Department, Bellvitge University Hospital-IDIBELL, C/Feixa Llarga s/n, 08907, Barcelona, Spain

${ }^{10}$ Departament de Psicobiologia i Metodologia de les Ciències de la Salut, Universitat Autònoma de Barcelona, 08193, Barcelona, Spain
}

Received: October 3, 2020 • Revised manuscript received: November 30, 2020; January 22, 2021; March 19,

2021 - Accepted: March 23, 2021

Published online: April 26, 2021

\section{ABSTRACT}

Background and aims: Increased delay discounting is associated with obesity and binge eating disorder (BED). Although BED and obesity frequently co-occur, the neural mechanisms underlying delay discounting in these conditions remain poorly understood. Methods: Thirtyfive women with obesity, including 10 participants with obesity and BED and 31 controls completed a monetary delay discounting task during functional magnetic resonance imaging. Results: We identified that increased discounting rates were associated with decreased activity in the left anterior insula in participants with obesity compared to controls when choosing immediate rewards over delayed rewards $\left(P_{\mathrm{FWE}}<0.05\right)$. An exploratory analysis comparing the BED subsample to the other groups did not detect significant differences. Discussion and conclusions: Our findings suggest decreased activity in the anterior insula may underlie heightened delay discounting in individuals with obesity, contributing the probability of choosing immediate rewards over delayed rewards based on emotional states. Future studies including larger, more diverse samples are required to confirm these effects. 


\section{KEYWORDS}

delay discounting, obesity, binge eating disorder, eating disorders, fMRI

\section{INTRODUCTION}

Delay discounting is a cognitive process describing how individuals value a reward to a lesser extent the farther into the future it is received; thereby devaluing delayed rewards as a function of time. Delay discounting differs substantially among individuals (Lempert, Steinglass, Pinto, Kable, \& Simpson, 2019), and high delay discounting is understood to reflect an inability to resist immediate reward over positive prospective outcomes. Steeper individual discounting rates (i.e., a preference for sooner, smaller reward) are associated with multiple clinical conditions characterized by deficits in impulse control (e.g., addictive disorders or gambling disorder) (Leeman \& Potenza, 2012; Steward, Mestre-Bach, Fernández-Aranda, et al., 2017; Steward et al., 2017).

In a manner similar to monetary reward, studies have suggested that individuals with obesity assign a higher value to the receipt of immediate food reward, even when satiated (Appelhans et al., 2011). Accordingly, delay discounting has been proposed as a potential psychological factor contributing to obesity (Amlung, Petker, Jackson, Balodis, \& Mackillop, 2016; Appelhans et al., 2011), as the choices of individuals with obesity might be swayed by a stronger preference for immediate rewards (i.e., unhealthy foods) over larger future rewards (i.e., improved health). Compared to healthy-weight controls, women with obesity tend to exhibit steeper discount patterns (Weller, Cook, Avsar, \& Cox, 2008). Likewise, studies have demonstrated high discounting rates to be positively associated with body mass index (BMI) (Jarmolowicz et al., 2014; Tang, ChrzanowskiSmith, Hutchinson, Kee, \& Hunter, 2019) and overeating (Appelhans et al., 2011). Steeper discount patterns could hamper efforts by individuals with obesity to reach a healthy weight as the tendency to choose immediate rewards is often incompatible with sustained dietary control and changes in eating habits (Maxwell, Gardiner, \& Loxton, 2020).

Delay discounting tasks performed during functional magnetic resonance imaging (fMRI) have provided insights into the neurobiological mechanisms underlying impulsive choices (Figner et al., 2010; Smith et al., 2016; Steward, Miranda-Olivos, Soriano-Mas, \& Fernández-Aranda, 2019). Multiple neural systems are believed to be involved in delay discounting, including those implicated in assigning value (the ventral striatum and ventromedial prefrontal cortex (vmPFC)), prospection (the posterior cingulate, precuneus, medial temporal lobe, and dorsomedial prefrontal cortex $(\mathrm{dmPFC})$ ), and executive control (anterior cingulate cortex (ACC) and dorsolateral prefrontal cortex (dlPFC)) (Lempert et al., 2019; Marco-Pallarés, Mohammadi, Samii, \& Münte, 2010; McClure, Laibson, Loewenstein, \& Cohen, 2004). Moreover, activity in the insula is believed to influence the probability of selecting smaller, sooner rewards over larger, delayed rewards based on affective states (Volkow \& Baler, 2015). Studies in healthy controls have found a bias to choose smaller immediate rewards over delayed reward to be associated with increased activity in the striatum, insula, and vmPFC (Koffarnus et al., 2017; Wittmann, Lovero, Lane, \& Paulus, 2010)' whereas increased activity, in the dlPFC and parietal regions, is associated with choosing larger delayed rewards (McClure et al., 2004; Wittmann, Leland, \& Paulus, 2007)

Neuroimaging studies have shown biases to immediate rewards in obesity to be associated with altered activity in prefrontal and parietal regions (Kishinevsky et al., 2012; Stoeckel, Murdaugh, Cox, Cook, \& Weller, 2013). Activity in these regions contributes to the inhibition of impulses and the planning future of actions by weighing possible longterm consequences (Chen, Papies, \& Barsalou, 2016). In the context of delay discounting, higher functional activity in the prefrontal cortex (PFC) and lower discounting rates have been associated with achieving (Kishinevsky et al., 2012; Weygandt et al., 2013) and maintaining weight loss (Weygandt et al., 2015). For instance, lower discounting rates associated with stronger activity in the dlPFC can predict, before a dietary intervention, successful weight loss at 12 weeks (Weygandt et al., 2013) and one-year following treatment (Weygandt et al., 2015). Relatedly, discounting rates have been observed to decrease after bariatric surgery in patients with morbid obesity (Budría et al., 2012). These studies demonstrate neural activity during decision-making can be a prognostic factor in dietary success and maintaining weight loss (Kishinevsky et al., 2012; Weygandt et al., 2013, 2015).

Binge eating disorder (BED) is characterized by distressful and frequent episodes of excessive food intake accompanied by a sense of loss of control (American Psychiatric Association, 2013). Although not all patients with BED have obesity, lack of control over food intake and increased binge frequency contribute to an $87 \%$ prevalence of obesity in individuals with BED (Villarejo et al., 2012). Some researchers support a framework wherein patients with BED represent a subgroup within a heterogeneous obesity phenotype (Hege et al., 2015; Jiménez-Murcia et al., 2019; Schag, Schönleber, Teufel, Zipfel, \& Giel, 2013). In pattern akin to behavioral addictions and substance abuse (Minhas et al., 2021; Steward, Mestre-Bach, Fernández-Aranda, et al., 2017), studies have also identified an association between higher discount rates and symptomatology in individuals with obesity and BED (Kekic et al., 2020; Manwaring, Green, Myerson, Strube, \& Wilfley, 2011). Women with BED and obesity have been found to present steeper discounting rates to food reward, compared to women with obesity without BED (non-BED) (Manwaring et al., 2011) and to monetary rewards, compared to control participants (Bartholdy et al., 2017; Steward, Mestre-Bach, VintróAlcaraz, et al., 2017). Moreover, theoretical models in obesity based on hedonic hunger and delay discounting have been designed to classify BED or non-BED according to individuals' scores (Manasse et al., 2015). In this study, high discounting rates and worse inhibitory control were associated with an increased probability of belonging to the BED group. 
While no study to date has examined delay discounting in individuals with BED using $\mathrm{PMRI}$, there is evidence to suggest that individuals with BED present distinct neural activation patterns during tasks involving inhibitory control in comparison to individuals with obesity without BED. For example, Balodis et al. (2013) found that individuals with BED presented diminished activity in the vmPFC, inferior frontal gyrus (IFG), and insula during incongruent trials on the Stroop task when compared to non-BED and controls. Voon (2015) suggests impulsive behaviors in individuals with BED are associated with greater cognitive impairment compared to those with non-BED obesity. In particular, impulsive behavior in $\mathrm{BED}$ is associated with greater behavioral inflexibility, compulsivity, and psychopathology compared to non-BED obesity (Voon, 2015).

In the study at hand, we examined monetary delay discounting during fMRI in women with obesity compared to controls. First, we aimed to investigate whether there were differences in neural activity associated with discounting rates between all participants with obesity and a control group. Second, in an exploratory sub-analysis, activation patterns during delay discounting in participants with BED were compared to participants with obesity and controls. We also sought to explore if differences in neural activity were associated with impulsive traits and eating disorder severity.

Based on prior literature, we hypothesized participants with obesity as a whole (OB-all) would exhibit higher discounting rates than controls and that this would be associated with reduced activation in the PFC, insula, and parietal regions, as well as increased activity in brain regions involved reward processing. Moreover, we hypothesized that BED participants would exhibit a differential activation pattern to non-BED participants with obesity during delay discounting. Considering the lack of fMRI studies in BED and the limited sample size of our BED group in this study, we have chosen not to further specify our hypotheses for this exploratory analysis.

\section{METHODS}

\section{Participants}

Sixtysix adult women (18-56 years of age) were included in the present study. Thirtyfive individuals belonged to the OBall group defined as having a BMI over 30 . Within the OBall group, 10 women had BED and 25 women did not have BED (non-BED). All women in the BED group met the Diagnostic and Statistical Manual of Mental Disorders (DSM-5) criteria for BED following a standardized structured interview conducted by clinical psychologists and psychiatrists. The BED and the non-BED groups were recruited from the Eating Disorders Unit and the Endocrinology and Nutrition Unit, respectively, at Bellvitge University Hospital in Barcelona, Spain. Participants in the nonBED group were patients seeking treatment for obesity and were required to a psychiatric screening in order to be considered a candidate for bariatric surgery. The BED and the non-BED groups were compared to 31 female controls $(\mathrm{BMI}=18-24.99)$, recruited via advertisements from the same University Hospital catchment area.

All participants underwent the Mini-International Neuropsychiatric Interview (M.I.N.I.) to assess the presence of a psychiatric disorder. In the case of controls, exclusion criteria were a lifetime history of an eating disorder, based on DSM-5 diagnostic criteria, having had obesity, and current diagnosis of psychiatric disorder. The study exclusion criteria for all participants were being male, the presence of an organic mental disorder or an intellectual disability, being pregnant or currently breastfeeding, and any contraindication for magnetic resonance imaging (MRI) scanning.

\section{Procedures}

Participants underwent assessments over two separate sessions. The first session consisted of collecting clinical and anthropometric measures (outside-scanner measures), while the MRI scanning was conducted at a second session.

\section{Measures}

Body composition. Initially, the participant height was measured by a stadiometer without wearing shoes. Then, this information was introduced in a leg-to-leg body composition analyzer using a Tanita BC-420MA (Tanita BC-420MA, Tanita Corp. Tokyo, Japan) to collect body composition variables and to obtain BMI. This instrument is a validated, non-invasive bioelectrical impedance analyzer that estimates body composition, considering age and sex.

Eating Disorders Inventory-2 (EDI-2). Eating disorder symptomatology was assessed via a validated Spanish version of the EDI-2 (Garner, Olmstead, \& Polivy, 1983). This is a self-report instrument to screen symptomatology related to eating disorders on a six-point Likert scale. It consists of 91 items and provides scores on 11 subscales: drive for thinness, body dissatisfaction, bulimia, ineffectiveness, perfectionism, interpersonal distrust, interoceptive awareness, maturity fears, asceticism, impulse regulation, and social insecurity. The sum of all subscales provides an eating disorder measure, which is considered a global scale of eating disorder severity. The internal consistency for the scale estimated through Cronbach's alpha was excellent $(\alpha=0.96)$.

Impulsivity measure (UPPS-P). This 59-item questionnaire is a multi-dimensional assessment of impulsivity using five distinct personality traits related to impulsive behavior. Specifically, it measures lack of perseverance, lack of premeditation, sensation seeking, negative urgency, and positive urgency. It contemplates acts or incidents that occurred during the last 6 months, scoring on a Likert scale of 1-5. The current study used the Spanish validation of the UPPS$\mathrm{P}$, which has demonstrated good reliability and validity (Verdejo-García, Lozano, Moya, Alcázar, \& Pérez-García, 2010). Internal consistency was between good $(\alpha=0.82$ for 
lack of premeditation) to excellent ( $\alpha=0.92$ for positive urgency).

MRI delay discounting paradigm. The items for our delay discounting task were initially designed by Kirby, Petry, and Bickel (1999) ' This questionnaire consists of 27 items evaluating intertemporal decision-making, and participant choice between two amounts of hypothetical monetary reward (e.g., between $€ 55$ "NOW" or $€ 81$ in 30 days). Each trial, considering its temporal gap and two rewards, is computed to provide a specific discounting rate $(k)$. Thus, the 27 discount rates derived from each item delineate a hyperbolic function rating from 0 to 0.25 . A higher $k$ indicates a greater devaluing of future rewards and a tendency to select smaller, immediate rewards.

The fMRI delay discounting task used in this study was adapted from prior work by Marco-Pallarès et al. (2010), and it was implemented using Presentation (Version 18.3, build 03.11.16, Neurobehavioral Systems, Albany, USA, www. neurobs.com). This task contained 4 runs with 27 trials included in each run. The total duration of each trial was 11 seconds beginning with the presentation of a cross fixation for 5 seconds to minimize carry-over effects, followed by the task block for 6 seconds. During the initial 3 seconds of the block, monetary choices were presented, and participants were required to give their responses during the final 3 seconds. Participants provided responses on an MRI-compatible button-box (Lumina 3G Controller, Cedrus Corporation), and the task was displayed via an angled mirror system using an MRI-compatible LCD screen (BOLD-screen 32, Cambridge Research Systems) located at the end of the scanner bore.

Individual discounting rates $(k)$ were calculated by identifying the switch-point of preference. In other words, the point when individuals change their preferences and begin to select delayed rewards over immediate rewards. Due to the hyperbolic distribution of discount parameter (Kirby \& Maraković, 1996), individual means were obtained by calculating the geometric mean across runs between trials, where the switch-point took place, and which produced choices consistent with individuals' $k$. In order to work with a linear distribution of individuals' $k$ rates, these were transformed using natural logarithmic (ln) transformation. Thus, $\ln (k)$ values range from -9 to 0 with 0 representing higher $k$ rates, and -9 representing lower $k$ rates.

\section{Data analysis}

Imaging data acquisition, preprocessing, and analysis. MRI data were obtained using a 3.0 Tesla MRI scanner (Intera, Philips Medical Systems, Eindhoven, Best, Netherlands) equipped with a 32-channel phased-array head coil. For the delay discounting task, 151 volumes per run were obtained with following parameters: repetition time of $2,000 \mathrm{~ms}$, echo time of $25 \mathrm{~ms}$, and a pulse angle of $90^{\circ}$; in a $24-\mathrm{cm}$ field of view (FOV); and an $80 \times 80$ pixel matrix delivering voxel sizes of $3 \times 3 \times 3 \mathrm{~mm}$ with no gap and 40 interleaved slices, parallel to the anterior-posterior commissure line. Each run had a duration of $4.95 \mathrm{~min}$. A high-resolution T1-weighted anatomical scan was also acquired to facilitate registration of the EPI data into standard space. A three-dimensional fast-spoiled gradient, an inversion-recovery sequence with 233 contiguous slices (repetition time, $10.43 \mathrm{~ms}$; echo time, $4.8 \mathrm{~ms}$; flip angle, $8^{\circ}$ ) in a 24 $\mathrm{cm}$ field of view with a $320 \times 320$ pixel matrix and isotropic voxel size of $0.75 \times 0.75 \times 0.75 \mathrm{~mm}$, was used.

fMRI preprocessing. All images were preprocessed using BrainWavelet Toolbox, which allows for the removal of high- and low-frequency artifacts from a time series by denoising the synchronized signal transients induced by head motion. The realigned functional sequences were coregistered to each participant's anatomical scan, which had been previously co-registered and normalized to the SPMT1 template. Further preprocessing took place using a pipeline in the CONN toolbox (version 19b, Massachusetts Institute of Technology, Cambridge, USA, http://www.nitrc. org/projects/conn) running on MATLAB R2019b. For each participant, preprocessing of functional data underwent the following steps: (1) motion correction, (2) slice-time correction, (3) ART-based identification of outlier scans for scrubbing, (4) spatial normalization applying the anatomical normalization parameters, which were then re-sliced to a $2 \mathrm{~mm}$ isotropic resolution in Montreal Neurological Institute (MNI) space, and (5) smoothing using a Gaussian filter with an $8 \mathrm{~mm}$ full width at half maximum (FWHM) kernel.

Next, denoising was applied to remove residual movement and physiological noise. Blood oxygen level-dependent (BOLD) time series were regressed against the six head motion parameters obtained from realignment and physiological noise deriving from white matter, cerebrospinal fluid (CSF), and global (BOLD) time-series were included as confounding factors. Last, temporal frequencies below 0.008 $\mathrm{Hz}$ or above $0.09 \mathrm{~Hz}$ were removed from the BOLD signal in order to remove additional artifacts.

First-level analyses. First-level (single subject) maps were estimated using statistical parametric mapping software (SPM 12, Welcome Department of Imaging Neuroscience, London, England; www.fil.ion.ucl.ac.uk/spm). A general linear model (GLM) was performed comparing the hemodynamic response during the 6 seconds of NOW and LATER choice blocks, across 4 runs. First-level activation was computed for each individual on NOW > LATER trials by convolving the time course of activation with the canonical hemodynamic response function (HRF). Heart rate frequency recorded during the fMRI paradigm was introduced as an individual regressor of hemodynamic response. The BOLD signal at each voxel was convolved using the SPM12 canonical HRF and a $128 \mathrm{~s}$ high-pass filter was applied.

Second-level analyses. First-level contrast images were then carried forward to a group-level random effects analysis, using a summary statistics approach (i.e., ANOVA). First, between-group comparisons (OB-all vs. controls or BED vs. non-BED vs. controls) examined differences in neural activation during NOW > LATER associated with discounting 
rates. Discounting rates represented by $\ln (k)$ were included as main variable of interest to explore brain activations during NOW > LATER trials. Given the significant differences between groups, age was introduced as a confounding variable. As a first step, a two-sample t-test was carried out comparing activation between participants in the OB-all group and the control group. Next, in order to investigate the distinctive brain activity within the obesity groups (BED and non-BED) in comparison to the control group, a one-way ANOVA was performed between these three groups. Significance thresholding from all derived differences in activation was set to satisfy a family-wise error (FWE) rate correction of $P_{\mathrm{FWE}}<$ 0.05 (spatial cluster extent) as well as, a threshold of $P<0.001$ as recommended by Woo, Krishnan, and Wager (2014) and Eklund, Nichols, and Knutsson (2016).

In order to explore the association between significant brain-derived differences between groups from the delay discounting task and clinical measures, derived peak activation eigenvalues from regions displaying significant differences were extracted.

Statistical analyses of non-imaging data. Statistical analyses of clinical measures and brain-derived eigenvalues were conducted with SPSS 23 (IBM Corp; Armonk, NY). Oneway ANOVA was employed for the global comparison of study variables between groups including post hoc pairwise comparisons via Scheffé's procedure. Effect sizes for mean differences were measured using Cohen's $d$ coefficient $(|d|>$ 0.2 was considered low-poor, $|d|>0.5$ mild-moderate, and $|d|>0.8$ high-large) (Kelley \& Preacher, 2012). The Finner method was also used to control Type-I error due to multiple comparisons (this method is included in FWE stepwise procedures) (Finner, 1993).

Associations within-groups were examined using Pearson's parametric correlations $(R)$. Due to the strong association for these parameters between significance test and sample size (low correlations estimated within large samples tend to provide significant results, whereas high correlations within low samples tend to provide non-significant values), the $R$ coefficients were interpreted attending to effect sizes $(|R|>$ 0.24 was considered mild-moderate and $|R|>0.37$ high-large; these thresholds corresponds to Cohen's-d of 0.20, 0.50, and 0.80 respectively) (Rosnow \& Rosenthal, 1996).

\section{Ethics}

The study procedures were carried out in accordance with the Declaration of Helsinki. The Clinical Research Ethics Committee of Bellvitge University Hospital (PR146/14) approved the study. Written informed consent was obtained from all participants before taking part in the study.

\section{RESULTS}

\section{Delay discounting and behavioral results}

Table 1 summarizes sociodemographic, anthropometric information, and psychological measures in the three groups.

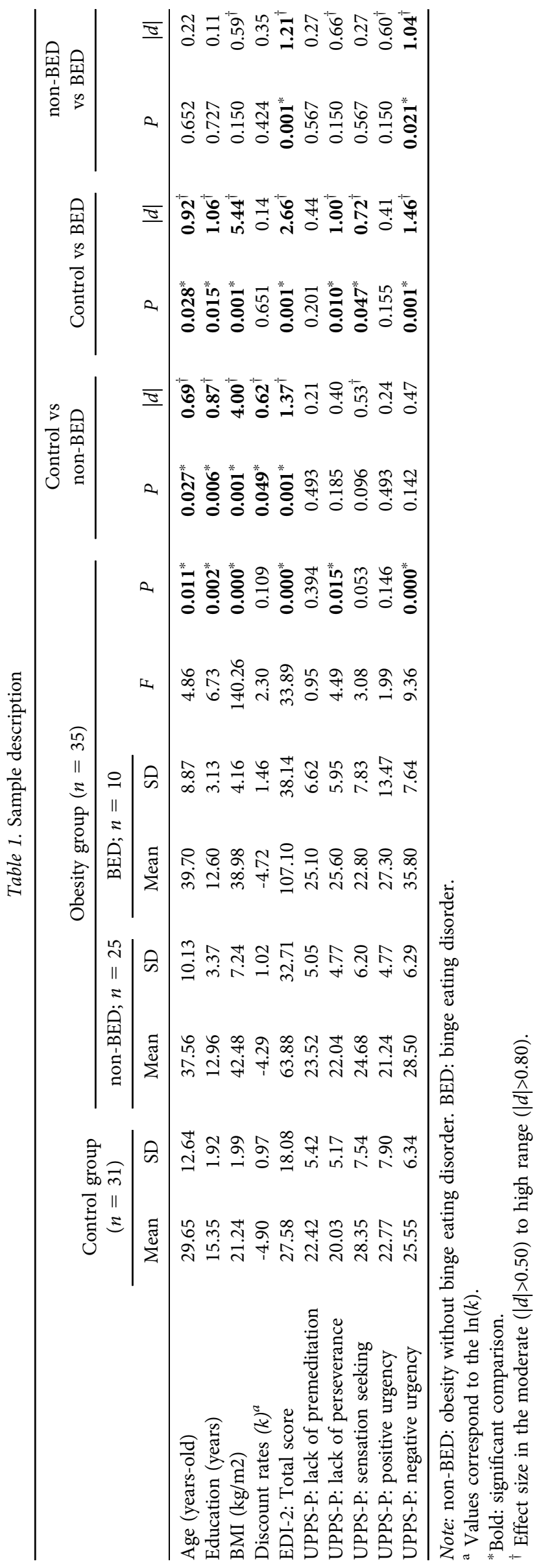



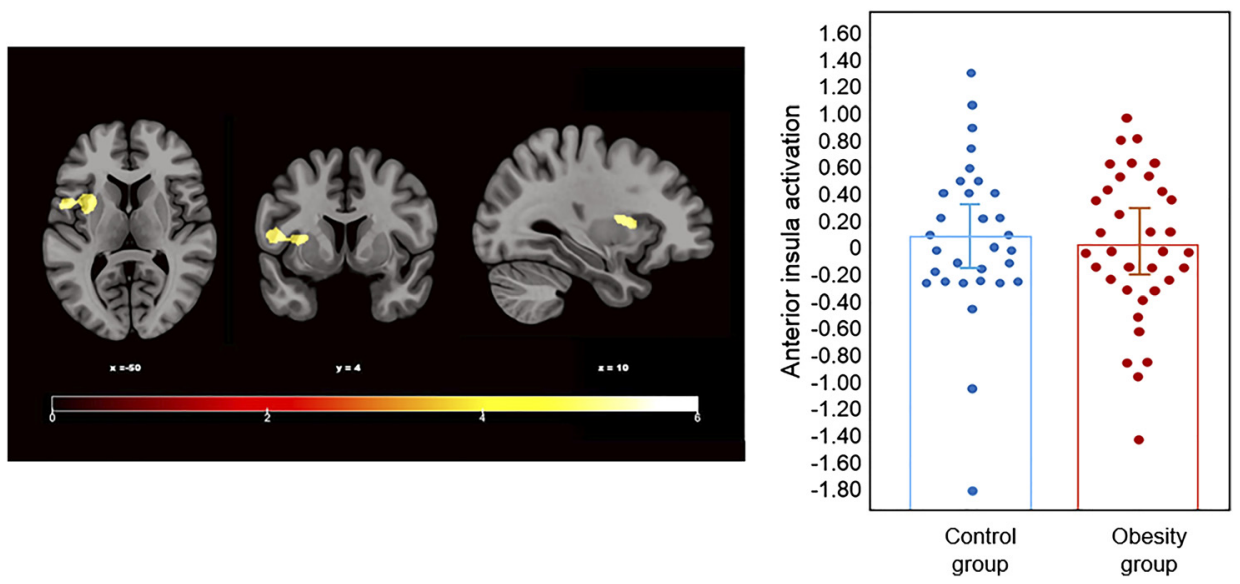

Fig. 1. The left panel depicts the identified increased activation in the left anterior insula associated with discount rates in the control group in comparison to the obesity group (OB-all) during NOW > LATER. Color bar represents t-values. Results are displayed at family-wise error (FWE) probability $\left(P_{\mathrm{FWE}}<0.05\right),(P<0.001)$, cluster-extent. The right panel depicts individual participant activation levels according to group

As expected, BMI was significantly different between the control group and clinical groups $(P<0.001)$. Likewise, there were significant differences in age $(P=0.006)$ between groups.

Concerning the behavioral delay discounting measure, the three groups did not significantly differ in discounting rates $(P=0.109$; Table 1$)$. However, a significance was observed when comparing the non-BED and control groups $(P=0.049$ : Table 1$)$. As would be expected, the BED group endorsed higher scores of eating disorder severity on the EDI-2 compared to control group $(P<0.001)$ and the nonBED group $(P<0.001)$. The BED group endorsed higher levels in lack of perseverance $(P=0.010)$ and negative urgency $(P=0.001)$ compared to controls on the UPPS-P. Likewise, the BED group had higher negative urgency levels $(P=0.021)$ compared to the non-BED group.

\section{Imaging data}

Group comparisons using independent sample t-tests during NOW $>$. LATER found that discounting rates were positively associated with decreased activation in the left anterior insula extending laterally through the temporal cortex in the OB-all group compared to the control group $\left(P_{\mathrm{FWE}}=0.029\right.$; Fig. 1; Table 2).

Group comparisons using a one-way ANOVA during NOW > LATER found no significant differences between the BED and the non-BED group, or the BED and the control group $\left(P_{\mathrm{FWE}}>0.05\right)$.

\section{DISCUSSION}

The present study investigated the neurobiological substrates of delay discounting in obesity with and without BED. We found a negative association between left anterior insula activation during NOW > LATER and individual discount
Table 2. Second-level fMRI delay discounting task results

\begin{tabular}{lcccc}
\hline $\begin{array}{l}\text { Contrast NOW }> \\
\text { LATER }\end{array}$ & Region & $\begin{array}{c}\text { MNI Coordinates } \\
(x, y, z)\end{array}$ & $\mathrm{ke}^{\mathrm{a}}$ & $t$ \\
\hline Controls $<$ OB-all & $\begin{array}{c}\text { Left anterior } \\
\text { insula }\end{array}$ & $-50 ; 4 ; 10$ & 232 & 4.87 \\
& & & & \\
\hline
\end{tabular}

Note: OB-all: obesity group (non-BED and BED). A general linear model (GLM) was performed comparing the hemodynamic response during the 6 seconds of NOW and LATER choice blocks of the delay discounting task, with NOW > LATER trials serving as our primary contrast of interest. Results satisfied a family-wise error $(\mathrm{FWE})$ probability $\left(P_{\mathrm{FWE}}<0.05\right)$ and $(P<0.001)$ clusterextent threshold.

MNI: Montreal Neurological Institute.

${ }^{a}$ Cluster extent in voxels.

rates in all participants with $\mathrm{OB}$ in comparison to controls. Contrary to our hypotheses, our exploratory analysis in BED patients did not identify differences in activation associated with discount rates during NOW > LATER. This lack of identified differences between groups is most likely due to a lack of power and are discussed in the limitations section.

The insula is understood to play a modulatory role in decision making by incorporating interoceptive and emotional processes during the deliberation between NOW vs LATER choices (Volkow \& Baler, 2015). In healthy controls, the anterior insula has been found to increase in activation when choosing immediate reward over delayed choices (Carter et al., 2010; Wittmann et al., 2010). Our finding associating higher delay discounting with decreased anterior insula activity suggests that individuals with obesity may experience impairments in integrating interoceptive signals with higher-level cognitive processing (Simmons et al., 2013; Zaki, Davis, \& Ochsner, 2012). Analogous results have been found in individuals with obesity and poor sleep quality (Martin et al., 2015). This study found diminished insula activity in individuals with obesity and poor sleep 
quality when making immediate and smaller monetary choices compared to a baseline condition. Similarly, recent research has identified decreased anterior insula activity during risky decisions following a loss in participants with obesity, which indicates that maladaptive signaling in the insula may underpin alterations in weighing the costs and benefits of decisions in obesity (Steward, Juaneda-Seguí, et al., 2019). Decreased modulation of putative executive function regions has also been found in women with obesity during difficult vs. easy trials of a delay discounting task (Stoeckel et al., 2013), suggesting that dysfunctional interactions between executive control and interceptive networks could contribute to excessive food intake (Steward, Menchon, et al., 2017; Syan et al., 2019). This compulsive eating pattern is associated with addictive-like eating behavior in obesity and is often defined using symptoms parallel to those of substance use disorders and behavioral addictions (Kakoschke, Aarts, \& Verdejo-García, 2019; Kekic et al., 2020). Likewise, addictive behaviors have been robustly associated with steep discounting to delayed reward (Volkow \& Baler, 2015), suggesting the presence of overlapping brain systems mediating appetitive and addictive behaviors (Volkow \& Baler, 2015).

It is plausible that reduced insula activation may partly underpin the emotionally driven impulsivity and decisionmaking impairments that characterize emotional eating in $\mathrm{OB}$ and BED (Steward \& Berner, 2020). In the case of patients with BED, a similar response to rewarding cues may manifest in the form of compulsive eating (Kakoschke et al., 2019). Food intake itself can become a source of distress as entrenched eating habits conflict with goals to normalize eating behaviors (Chao et al., 2016; Munsch, Meyer, Quartier, \& Wilhelm, 2012). It should be noted that the BED group also showed higher negative urgency scores than the non-BED and control groups. For individuals with $\mathrm{BED}$, negative urgency is often associated with excessive eating, which is used to alleviate negative emotions (Aloi et al., 2020; Lavender \& Mitchell, 2015; Munsch et al., 2012). However, any inferences emerging from these preliminary results must be interpreted with caution given the limited sample size featured in this study. As such, future studies with larger samples are required to confirm this effect.

Last, in contrast to other studies, our results did not identify significant behavioral differences when comparing discounting rates between all participants with obesity and controls and only a marginal significance was found when comparing the non-BED group to controls. Our findings are partly supported by a systematic review by McClelland et al. (2016), which identified mixed results when comparing individuals with obesity to controls. While most studies identified high discount rates in individuals with obesity compared to controls, a relevant proportion of studies did not report differences (McClelland et al., 2016). The authors underscored the importance of examining whether the use of hypothetical (vs. real) monetary rewards can influence discounting rates. Similarly, food vs non-food rewards are known to influence delay discounting in individuals with obesity and BED and controls (Manwaring et al., 2011; McClelland et al., 2016).

Although this study has its strengths, some limitations should be considered when interpreting its results. First, our sample does not fully represent the general population with obesity as our participants were recruited from a hospital setting and seeking treatment (i.e., bariatric surgery or psychotherapy). Second, our study cannot make inferences regarding causality due to its cross-sectional design. Third, this study only recruited women and future studies should aim to include larger and diverse samples, especially in patients with BED. It would be of interest to examine whether higher discount rates are a risk factor for compulsive overeating and whether interventions targeted at orienting individuals with BED to increase the value of future rewards could produce a meaningful decrease in binge eating behaviors (Juarascio, Manasse, Espel, Kerrigan, \& Forman, 2015). Likewise, it would have been of interest to consider hormonal factors (e.g., estrogens levels), which are known to modulate reward response (Diekhof, 2015). Finally, our sample size was limited and our failure to identify significant differences between groups is likely due to a lack of statistical power.

\section{CONCLUSIONS}

Our findings provide evidence of alterations in anterior insula function in individuals with obesity and BED during delay discounting. Future studies with larger samples and using delay discounting paradigms may shed light as to why a subset of individuals with obesity may be prone to binge eating episodes. Likewise, it would be of interest for future studies to integrate both general and food-specific tasks with neuroimaging in order to further delineate the neural circuitry that contributes to BED (Berner et al., 2017).

Funding sources: This manuscript and research were supported by grants from the Ministerio de Economía y Competitividad (PSI2015-68701-R), Ministerio de Sanidad, Servicios Sociales e Igualdad (PR338/17), Instituto de Salud Carlos III (ISCIII) (FIS PI14/00290, PI17/01167 and PI20/ 132) and co-funded by FEDER funds/European Regional Development Fund (ERDF), a way to build Europe. CIBERObn and CIBERSAM are both initiatives of ISCIII. This research was supported by a PNSD (PR338/17-MSSSI) grant. Additional support was received from EU Grant Eat2beNice (H2020-SFS-2016-2; Ref 728018). TS is supported by a NHMRC/MRFF Investigator Grant (MRF1193736), a BBRF Young Investigator Grant, and a University of Melbourne McKenzie Fellowship. GMB is supported by a postdoctoral grant from FUNCIVA. We thank CERCA/the Generalitat de Catalunya for institutional support. The funders had no role in the study design, data collection, and analysis, decision to publish, or preparation of the manuscript. 
Author's contributions: R.M-O.: Conceptualization, Investigation, Methodology, Project administration, Data curation, Writing-original draft; T.S.: Conceptualization, Investigation, Methodology, Project administration, Data curation, Writing-original draft; I.M.-Z.: Formal analysis, Investigation, Methodology, Data curation; G.M.-B.: Investigation, Project administration, Data curation; A.S.-J.: Methodology, Data curation; S.J.-M.: Funding acquisition; J.A.F.-F.: Funding acquisition; N.V. (Nuria Vilarrasa): Resources; M.V.d.l.H.: Resources; N.C.: Resources; N.V. (Nuria Virgili): Resources; R.L.-U.: Resources; J.M.M.: Funding acquisition; C.S.-M.: Conceptualization, Investigation, Project administration, Data curation, Writing - original draft; F.F.-A.: Conceptualization, Investigation, Project administration; Funding acquisition, Writing-original draft.

Conflict of Interest: FF-A declares having received consultation honoraries from Novo Nordisk and editorial honoraries from Wiley. The rest of the authors declare no conflict of interest.

\section{REFERENCES}

Aloi, M., Rania, M., Carbone, E. A., Calabrò, G., Caroleo, M., Carcione, A., et al. (2020). The role of self-monitoring metacognition sub-function and negative urgency related to binge severity. Eur Eat Disorders Rev, 28(5), 580-586.

American Psychiatric Association. (2013). DSM-5 diagnostic classification. In Diagnostic and statistical manual of mental disorders. American Psychiatric Association. https://doi.org/10 1176/appi.books.9780890425596.x00diagnosticclassification.

Amlung, M., Petker, T., Jackson, J., Balodis, I., \& Mackillop, J. (2016). Steep discounting of delayed monetary and food rewards in obesity: A meta-analysis. Psychological Medicine, 46(11), 2423-2434. https://doi.org/10.1017/S00332917160 00866.

Appelhans, B. M., Woolf, K., Pagoto, S. L., Schneider, K. L., Whited, M. C., \& Liebman, R. (2011). Inhibiting food reward: Delay discounting, food reward sensitivity, and palatable food intake in overweight and obese women. Obesity, 19(11), 2175-2182. https://doi.org/10.1038/oby.2011.57.

Balodis, I. M., Kober, H., Worhunsky, P. D., White, M. A., Stevens, M. C., Pearlson, G. D., et al. (2013). Monetary reward processing in obese individuals with and without binge eating disorder. Biological Psychiatry, 73(9), 877-886. https://doi.org/ 10.1016/j.biopsych.2013.01.014.

Bartholdy, S., Rennalls, S., Danby, H., Jacques, C., Campbell, I. C., Schmidt, U., et al. (2017). Temporal discounting and the tendency to delay gratification across the eating disorder spectrum. European Eating Disorders Review, 25(5), 344-350. https://doi. org/10.1002/erv.2513.

Berner, L. A., Winter, S. R., Matheson, B. E., Benson, L., \& Lowe, M. R. (2017). Behind binge eating: A review of food-specific adaptations of neurocognitive and neuroimaging tasks. Physiology \& Behavior, 176, 59-70. https://doi.org/10.1016/j.physbeh.2017. 03.037 .
Budría, S., Lacomba, J. A., Lagos, F., \& Swedberg, P. (2012). When obese people are more patient than non-obese people. A study of post-surgery individuals in a weight loss association. Revista Internacional de Sociología, 70(Extra_1), 83-98. https://doi.org/ 10.3989/ris.2011.05.04.

Carter, R. M. K., Meyer, J. R., \& Huettel, S. A. (2010). Functional neuroimaging of Intertemporal choice models: A review. Journal of Neuroscience, Psychology, and Economics, 3(1), 27-45. https://doi.org/10.1037/a0018046.

Chao, A. M., Wadden, T. A., Faulconbridge, L. F., Sarwer, D. B., Webb, V. L., Shaw, J. A., et al. (2016). Binge-eating disorder and the outcome of bariatric surgery in a prospective, observational study: Two-year results. Obesity, 24(11), 2327-2333. https://doi. org/10.1002/oby.21648.

Chen, J., Papies, E. K., \& Barsalou, L. W. (2016). A core eating network and its modulations underlie diverse eating phenomena. Brain and Cognition, 110, 20-42. https://doi.org/10.1016/j. bandc.2016.04.004.

Diekhof, E. K. (2015). Be quick about it. Endogenous estradiol level, menstrual cycle phase and trait impulsiveness predict impulsive choice in the context of reward acquisition. Hormones and Behavior, 74, 186-193. https://doi.org/10.1016/ j.yhbeh.2015.06.001.

Eklund, A., Nichols, T. E., \& Knutsson, H. (2016). Cluster failure: Why fMRI inferences for spatial extent have inflated falsepositive rates. Proceedings of the National Academy of Sciences of the United States of America, 113(28), 7900-7905. https://doi. org/10.1073/pnas.1602413113.

Figner, B., Knoch, D., Johnson, E. J., Krosch, A. R., Lisanby, S. H., Fehr, E., et al. (2010). Lateral prefrontal cortex and self-control in intertemporal choice. Nature Neuroscience, 13(5), 538-539. https://doi.org/10.1038/nn.2516.

Finner, H. (1993). On a Monotonicity problem in step-down multiple test procedures. Journal of the American Statistical Association, 88(423), 920. https://doi.org/10.2307/2290782.

Garner, D. M., Olmstead, M. P., \& Polivy, J. (1983). Development and validation of a multidimensional eating disorder inventory for anorexia nervosa and bulimia. International Journal of Eating Disorders, 2(2). https://doi.org/10.1002/1098108X(198321)2:2<15::AID-EAT2260020203>3.0.CO;2-6.

Hege, M. A., Stingl, K. T., Kullmann, S., Schag, K., Giel, K. E., Zipfel, S., et al. (2015). Attentional impulsivity in binge eating disorder modulates response inhibition performance and frontal brain networks. International Journal of Obesity, 39(2), 353-360. https://doi.org/10.1038/ijo.2014.99.

Jarmolowicz, D. P., Cherry, J. B. C., Reed, D. D., Bruce, J. M., Crespi, J. M., Lusk, J. L., et al. (2014). Robust relation between temporal discounting rates and body mass. Appetite, 78, 63-67. https://doi.org/10.1016/j.appet.2014.02.013.

Jiménez-Murcia, S., Agüera, Z., Paslakis, G., Munguia, L., Granero, R., Sánchez-González, J., et al. (2019). Food addiction in eating disorders and obesity: Analysis of clusters and implications for treatment. Nutrients, 11(11). https://doi.org/10.3390/ nu11112633.

Juarascio, A. S., Manasse, S. M., Espel, H. M., Kerrigan, S. G., \& Forman, E. M. (2015). Could training executive function improve treatment outcomes for eating disorders? Appetite, 90, 187-193. https://doi.org/10.1016/j.appet.2015.03.013. 
Kakoschke, N., Aarts, E., \& Verdejo-García, A. (2019). The cognitive drivers of compulsive eating behavior. In Frontiers in behavioral neuroscience (Vol. 12). Frontiers Media S.A. https:// doi.org/10.3389/fnbeh.2018.00338.

Kekic, M., McClelland, J., Bartholdy, S., Chamali, R., Campbell, I. C., \& Schmidt, U. (2020). Bad things come to those who do not wait: Temporal discounting is associated with compulsive overeating, eating disorder psychopathology and food addiction. Frontiers in Psychiatry, 10(January), 1-9. https://doi.org/ 10.3389/fpsyt.2019.00978.

Kelley, K., \& Preacher, K. J. (2012). On effect size. Psychological Methods, 17(2), 137-152. https://doi.org/10.1037/a0028086.

Kirby, K. N., \& Maraković, N. N. (1996). Delay-discounting probabilistic rewards: Rates decrease as amounts increase. Psychonomic Bulletin \& Review, 3(1), 100-104. https://doi.org/ 10.3758/BF03210748.

Kirby, K. N., Petry, N. M., \& Bickel, W. K. (1999). Heroin addicts have higher discount rates for delayed rewards than non-drugusing controls. Journal of Experimental Psychology: General, 128(1), 78-87. https://doi.org/10.1037/0096-3445.128.1.78.

Kishinevsky, F. I., Cox, J. E., Murdaugh, D. L., Stoeckel, L. E., Cook, E. W., Weller, R. E. (2012). FMRI reactivity on a delay discounting task predicts weight gain in obese women. Appetite, 58(2), 582-592. https://doi.org/10.1016/j.appet.2011.11.029.

Koffarnus, M. N., Deshpande, H. U., Lisinski, J. M., Eklund, A., Bickel, W. K., \& LaConte, S. M. (2017). An adaptive, individualized fMRI delay discounting procedure to increase flexibility and optimize scanner time. NeuroImage, 161, 56-66. https:// doi.org/10.1016/j.neuroimage.2017.08.024.

Lavender, J. M., \& Mitchell, J. E. (2015). Eating disorders and their relationship to impulsivity. Current Treatment Options in Psychiatry, 2(4), 394-401. https://doi.org/10.1007/s40501-0150061-6.

Leeman, R. F., \& Potenza, M. N. (2012). Similarities and differences between pathological gambling and substance use disorders: A focus on impulsivity and compulsivity. Psychopharmacology, 219(2), 469-490. https://doi.org/10.1007/s00213-011-2550-7.

Lempert, K. M., Steinglass, J. E., Pinto, A., Kable, J. W., \& Simpson, H. B. (2019). Can delay discounting deliver on the promise of RDoC? Psychological Medicine, 49(2), 190-199. https://doi.org/ 10.1017/S0033291718001770.

Manasse, S. M., Espel, H. M., Forman, E. M., Ruocco, A. C., Juarascio, A. S., Butryn, M. L., et al. (2015). The independent and interacting effects of hedonic hunger and executive function on binge eating. Appetite, 89, 16-21. https://doi.org/10. 1016/j.appet.2015.01.013.

Manwaring, J. L., Green, L., Myerson, J., Strube, M. J., \& Wilfley, D. E. (2011). Discounting of various types of rewards by women with and without binge eating disorder: Evidence for general rather than specific differences. Psychological Record, 61(4), 561-582. https://doi.org/10.1007/BF03395777.

Marco-Pallarés, J., Mohammadi, B., Samii, A., \& Münte, T. F. (2010). Brain activations reflect individual discount rates in intertemporal choice. Brain Research, 1320, 123-129. https:// doi.org/10.1016/j.brainres.2010.01.025.

Martin, L. E., Pollack, L., McCune, A., Schulte, E., Savage, C. R., \& Lundgren, J. D. (2015). Comparison of obese adults with poor versus good sleep quality during a functional neuroimaging delay discounting task: A pilot study. Psychiatry Research Neuroimaging, 234(1), 90-95. https://doi.org/10.1016/j. pscychresns.2015.08.011.

Maxwell, A. L., Gardiner, E., \& Loxton, N. J. (2020). Investigating the relationship between reward sensitivity, impulsivity, and food addiction: A systematic review. European Eating Disorders Review, 28(4), 368-384. https://doi.org/10.1002/erv.2732.

McClelland, J., Dalton, B., Kekic, M., Bartholdy, S., Campbell, I. C., \& Schmidt, U. (2016). A systematic review of temporal discounting in eating disorders and obesity: Behavioural and neuroimaging findings. Neuroscience \& Biobehavioral Reviews, 71, 506-528. https://doi.org/10.1016/j.neubiorev.2016.09.024.

McClure, S. M., Laibson, D. I., Loewenstein, G., \& Cohen, J. D. (2004). Separate neural systems value immediate and delayed monetary rewards. Science, 306(5695), 503-507. https://doi.org/ 10.1126/science.1100907.

Minhas, M., Murphy, C. M., Balodis, I. M., Acuff, S. F., Buscemi, J., Murphy, J. G., et al. (2021). Multidimensional elements of impulsivity as shared and unique risk factors for food addiction and alcohol misuse. Appetite, 159, 105052. https://doi.org/10. 1016/j.appet.2020.105052.

Munsch, S., Meyer, A. H., Quartier, V., \& Wilhelm, F. H. (2012). Binge eating in binge eating disorder: A breakdown of emotion regulatory process? Psychiatry Research, 195(3), 118-124. https://doi.org/10.1016/j.psychres.2011.07.016.

Rosnow, R. L., \& Rosenthal, R. (1996). Computing contrasts, effect sizes, and counternulls on other people's published data: General procedures for research consumers. Psychological Methods, 1(4). https://doi.org/10.1037/1082-989X.1.4.331.

Schag, K., Schönleber, J., Teufel, M., Zipfel, S., \& Giel, K. E. (2013). Food-related impulsivity in obesity and Binge Eating Disorder a systematic review. Obesity Reviews, 14(6), 477-495. https:// doi.org/10.1111/obr.12017.

Simmons, W. K., Rapuano, K. M., Kallman, S. J., Ingeholm, J. E., Miller, B., \& Gotts, S. J., et al. (2013). Category-specific integration of homeostatic signals in caudal but not rostral human insula. Nature Neuroscience, 16(11), 1551-1552. https://doi.org/ 10.1038/nn.3535.

Smith, C. T., Wallace, D. L., Dang, L. C., Aarts, E., Jagust, W. J., D’Esposito, M., et al. (2016). Modulation of impulsivity and reward sensitivity in intertemporal choice by striatal and midbrain dopamine synthesis in healthy adults. Journal of Neurophysiology, 115(3), 1146-1156. https://doi.org/10.1152/jn. 00261.2015.

Steward, T., \& Berner, L. A. (2020). The neurobiological basis of executive function alterations in binge eating populations. In Binge eating: A transdiagnostic psychopathology (pp. 137-152). Springer International Publishing. https://doi.org/10.1007/9783-030-43562-2_10.

Steward, T., Juaneda-Seguí, A., Mestre-Bach, G., Martínez-Zalacaín, I., Vilarrasa, N., Jiménez-Murcia, S., et al. (2019). What difference does it make? Risk-taking behavior in obesity after a loss is associated with decreased ventromedial prefrontal cortex activity. Journal of Clinical Medicine, 8(10), 1551. https://doi. org/10.3390/jcm8101551.

Steward, T., Menchon, J. M., Jiménez-Murcia, S., Soriano-Mas, C., \& Fernandez-Aranda, F. (2017). Neural network alterations across eating disorders: A narrative review of fMRI studies. 
Current Neuropharmacology, 16(8), 1150-1163. https://doi.org/ 10.2174/1570159x15666171017111532.

Steward, T., Mestre-Bach, G., Fernández-Aranda, F., Granero, R., Perales, J. C., Navas, J. F., et al. (2017). Delay discounting and impulsivity traits in young and older gambling disorder patients. Addictive Behaviors, 71, 96-103. https://doi.org/10.1016/ j.addbeh.2017.03.001.

Steward, T., Mestre-Bach, G., Vintró-Alcaraz, C., Agüera, Z., Jiménez-Murcia, S., Granero, R., et al. (2017). Delay discounting of reward and impulsivity in eating disorders: From anorexia nervosa to binge eating disorder. European Eating Disorders Review, 25(6), 601-606. https://doi.org/10.1002/erv.2543.

Steward, T., Miranda-Olivos, R., Soriano-Mas, C., \& FernándezAranda, F. (2019). Neuroendocrinological mechanisms underlying impulsive and compulsive behaviors in obesity: A narrative review of fMRI studies. Reviews in Endocrine \& Metabolic Disorders, 20(3), 263-272. https://doi.org/10.1007/s11154-01909515-x.

Steward, T., Mestre-Bach, G., Fernández-Aranda, F., Granero, R., Perales, J. C., Navas, J. F., et al. (2017). Delay discounting and impulsivity traits in young and older gambling disorder patients. Addictive Behaviors, 71, 96-103. https://doi.org/10.1016/ j.addbeh.2017.03.001. 28288442.

Stoeckel, L. E., Murdaugh, D. L., Cox, J. E., Cook, E. W., \& Weller, R. E. (2013). Greater impulsivity is associated with decreased brain activation in obese women during a delay discounting task. Brain Imaging and Behavior, 7(2), 116-128. https://doi. org/10.1007/s11682-012-9201-4.

Syan, S. K., Owens, M. M., Goodman, B., Epstein, L. H., Meyre, D., Sweet, L. H., et al. (2019). Deficits in executive function and suppression of default mode network in obesity. NeuroImage: Clinical, 24, 102015. https://doi.org/10.1016/j.nicl.2019.102015.

Tang, J., Chrzanowski-Smith, O. J., Hutchinson, G., Kee, F., \& Hunter, R. F. (2019). Relationship between monetary delay discounting and obesity: A systematic review and meta-regression. International Journal of Obesity, 43(6), 1135-1146. Nature Publishing Group. https://doi.org/10.1038/s41366-018-0265-0.

Verdejo-García, A., Lozano, O., Moya, M., Alcázar, M. A., \& PérezGarcía, M. (2010). Psychometric properties of a Spanish version of the UPPS-P impulsive behavior scale: Reliability, validity and association with trait and cognitive impulsivity. Journal of Personality Assessment, 92(1), 70-77. https:/doi.org/10.1080/ 00223890903382369
Villarejo, C., Fernández-Aranda, F., Jiménez-Murcia, S., PeñasLledó, E., Granero, R., Penelo, E., et al. (2012). Lifetime obesity in patients with eating disorders: Increasing prevalence, clinical and personality correlates. European Eating Disorders Review, 20(3), 250-254. https://doi.org/10.1002/erv.2166.

Volkow, N. D., \& Baler, R. D. (2015). NOW vs LATER brain circuits: Implications for obesity and addiction. Trends in Neurosciences, 38(6), 345-352. https://doi.org/10.1016/j.tins.2015. 04.002 .

Voon, V. (2015). Cognitive biases in binge eating disorder: The hijacking of decision making. In CNS spectrums 20(6), 566-573. Cambridge University Press. https://doi.org/10.1017/ S1092852915000681.

Weller, R. E., Cook, E. W., Avsar, K. B., \& Cox, J. E. (2008). Obese women show greater delay discounting than healthy-weight women. Appetite, 51(3), 563-569. https://doi.org/10.1016/j. appet.2008.04.010.

Weygandt, M., Mai, K., Dommes, E., Leupelt, V., Hackmack, K., Kahnt, T., et al. (2013). The role of neural impulse control mechanisms for dietary success in obesity. NeuroImage, 83, 669-678. https://doi.org/10.1016/j.neuroimage.2013.07.028.

Weygandt, M., Mai, K., Dommes, E., Ritter, K., Leupelt, V., Spranger, J., et al. (2015). Impulse control in the dorsolateral prefrontal cortex counteracts post-diet weight regain in obesity. NeuroImage, 109, 318-327. https://doi.org/10.1016/J. NEUROIMAGE.2014.12.073.

Wittmann, M., Leland, D. S., \& Paulus, M. P. (2007). Time and decision making: Differential contribution of the posterior insular cortex and the striatum during a delay discounting task. Experimental Brain Research, 179(4), 643-653. https://doi.org/ 10.1007/s00221-006-0822-y.

Wittmann, M., Lovero, K. L., Lane, S. D., \& Paulus, M. P. (2010). Now or later? Striatum and insula activation to immediate versus delayed rewards. Journal of Neuroscience, Psychology, and Economics, 3(1), 15-26. https://doi.org/10.1037/a0017252.

Woo, C. W., Krishnan, A., \& Wager, T. D. (2014). Cluster-extent based thresholding in fMRI analyses: Pitfalls and recommendations. NeuroImage, 91, 412-419. https://doi.org/10.1016/j. neuroimage.2013.12.058.

Zaki, J., Davis, J. I., \& Ochsner, K. N. (2012). Overlapping activity in anterior insula during interoception and emotional experience. NeuroImage, 62(1), 493-499. https://doi.org/10.1016/j. neuroimage.2012.05.012. 